\title{
Síntomas ansioso-depresivos en niños y su relación con los estilos educativos de los padres
}

\section{Anxiety and depressive symptoms in children and their relationship with parents' educational style}

Fecha de recepción: 19-07-2017

Fecha de aceptación: 9-11-2017
David Pineda

Universidad Nacional de Educación a Distancia, Facultad de Psicología.

Rosa María Valiente

Universidad Nacional de Educación a Distancia, Facultad de Psicología.

Ana Martínez-Martínez

Thinkids Psicología, Elche

Paloma Chorot

Universidad Nacional de Educación a Distancia, Facultad de Psicología.

Bonifacio Sandín.

Universidad Nacional de Educación a Distancia, Facultad de Psicología.

\section{resumen/alsstract:}

La evidencia reciente indica que la psicopatología del niño está determinada por múltiples factores, tanto personales como familiares y sociales. Entre los factores familiares cabe destacar la influencia de las prácticas de crianza de los progenitores. Sin embargo, la relación entre las prácticas educativas de los padres y los síntomas de ansiedad y depresión de los niños, en función de sexo de los padres ha sido menos estudiada. Los objetivos de esta investigación han sido examinar la relación entre las prácticas educativas parentales y los síntomas interiorizados de los niños, así como las posibles diferencias en función del sexo de los progenitores; un segundo objetivo ha sido explorar el papel que la edad y el sexo de los niños pueden desempeñar en estas relaciones. Estas cuestiones fueron examinadas en una muestra por conveniencia de 211 niños con edades comprendidas entre los 7 y los 12 años. En general, los resultados obtenidos apoyan la hipótesis de las diferencias en función del sexo de los progenitores. Las prácticas de crianza de la madre parecen relacionarse con una mayor probabilidad de sufrir trastornos ansioso-depresivos, mientras que el estilo del padre podría jugar un papel protector frente a estas patologías. Se discuten los resultados encontrados en base a la literatura existente y se proponen algunos aspectos que debería abordar la investigación futura.

Recent evidence indicates that child psychopathology is influenced by personal, family and social factors. Family factors include the influence of parenting child-rearing behaviors. However, relationships between parental rearing behaviors and children's anxiety and depression symptoms based on parental gender has been less studied. An objective of this study was to examine relationship between parental rearing behaviors and internalizing symptoms of children, as well as possible differences associated to the gender of parents. A second aim was to explore the role of age and gender of children on these relationships. These goals were examined in a sample of 211 children aged 7 to 12 years. Results support that mother-rearing behaviors appear to be related to an increased likelihood of anxiety-depressive symptoms, while the father's style may play a protective effect. We discuss the implications of results and propose suggestions for future research

\section{palabras clave/keywords:}

Estilos parentales, ansiedad, depresión, niños

Parental styles, Anxiety, depression, children. 
La evidencia reciente indica que la psicopatología del niño está determinada por múltiples factores, tanto personales como familiares y sociales. Entre los personales se incluyen los factores de riesgo (p.ej., inhibición conductual, afectividad negativa, sensibilidad a la ansiedad) (Ordóñez-Ortega, Espinosa-Fernández, García-López, y Muela-Martínez, 2013; Valiente, Sandín, y Chorot, 2012) o protección (p.ej., resiliencia) (Rutter, 2003). Los factores familiares incluyen tanto factores relacionados específicamente con los padres (p.ej., los trastornos mentales, el consumo de sustancias, etc.) (Vostanis et al., 2006; Zlomke, Lamport, Bauman, Garland, y Talbot, 2014), como aspectos referidos a las interacciones entre los padres y el hijo, tales como el apego, los estilos de crianza o las prácticas parentales (Romano, Tremblay, Boulerice, y Swisher, 2005). La influencia de las prácticas de crianza de los padres en el comportamiento de sus hijos ha sido ampliamente estudiada desde mediados del siglo pasado. Estos primeros trabajos con un enfoque más tipológico, se centraron en analizar los componentes de los estilos educativos y su clasificación (Baumrind, 1968; Maccoby y Martin, 1983). Posteriormente estos trabajos se han ido complementando con otras investigaciones que conciben el estilo educativo como un continuo en una serie de dimensiones. Hoy en día se concibe el estilo educativo desde un modelo bidireccional que entiende que el niño no es un mero receptor pasivo de los estilos educativos, y que las características personales del niño también influyen en el trato paterno, y por tanto el estilo educativo es el resultado de la interacción entre las características de los padres y las de los hijos (Tur, Mestre, y del Barrio, 2014). Existe abundante evidencia acerca de la relación entre los estilos de crianza y los síntomas emocionales en niños, sin embargo la relación de esta asociación con el sexo del progenitor ha sido menos estudiada.

Los objetivos de este trabajo han sido examinar la relación entre las prácticas educativas parentales y los síntomas interiorizados de los niños y si existen diferencias en función del sexo de los progenitores; un segundo objetivo ha sido explorar el papel que la edad y el sexo de los niños pueden desempeñar en estas relaciones.

Se espera que las prácticas de crianza más eficaces (implicación parental, crianza positiva y disciplina apropiada) predigan unos menores niveles de ansiedad y depresión, mientras que aquellas prácticas de crianza menos eficaces (disciplina inconsistente, pobre supervisión y disciplina severa) estarán relacionadas con mayores niveles de síntomas de ansiedad y depresión. Se prevé que el sexo de los progenitores tenga un efecto mediador en estas relaciones. Así mismo, se espera que la edad y el sexo de los niños mediará la relación entre las prácticas parentales y la sintomatología ansioso-depresiva de los niños.

\section{Método}

\section{Muestra}

La muestra estuvo compuesta por 118 chicos $(51.81 \%)$ y 93 chicas (48.18\%), que representan un total de 211 niños de edades comprendidas entre los 7 y los 12 años, con una media de edad de $9.62(\mathrm{DT}=1.05)$. El nivel educativo abarcaba desde $3^{\circ}$ a $6^{\circ}$ de primaria. El 88\% convivía con ambos padres, un $10 \%$ solo con uno de ellos y $2 \%$ restante tenía otro tipo de organización familiar. 


\section{Procedimiento}

Los sujetos pertenecían a un colegio concertado de la provincia de Alicante. Se recabó el consentimiento informado tanto de los padres como de los niños, informándoles de que su participación en la investigación era libre y anónima y en cualquier momento podrían dar por finalizada su participación, si así lo deseaban. Los sujetos cumplimentaron una batería de pruebas como parte de una investigación mucho más amplia. El tiempo empleado para completar todas las pruebas osciló entre los 45 y los 60 minutos dividiendo el protocolo en dos sesiones cuando fue necesario. Los cuestionarios se completaron de forma grupal a través de la plataforma online del grupo de investigación.

\section{Instrumentos}

Revised Child Anxiety and Depression Scale -30 (RCADS-30). La Revised Child Anxiety and Depression Scale (RCADS; Chorpita, Yim, Moffit, Umemoto, y Francis, 2000) fue desarrollada por el grupo de Chorpita como una revisión del The Spence Children's Anxiety Scale (SCAS; Spence, 1997, 1998). Formada por un total de 47 ítems clasificados en seis subescalas: ansiedad de separación (7 ítems), fobia social (9 ítems), trastorno de ansiedad generalizada ( 6 ítems), trastorno de pánico ( 9 ítems), trastorno obsesivo compulsivo (6 ítems) y trastorno depresivo mayor (10 ítems). La versión española de prueba mantiene la estructura y el número de ítems de la versión original (Sandín, Valiente, y Chorot, 2009). Posteriormente con estudios basados en análisis factoriales se desarrolló una versión reducida de la escala con tan solo 30 ítems, respetando la estructura original y con propiedades psicométricas similares a la versión de 47 ítems (Sandin, Chorot, Valiente, y Chorpita, 2010). Recientes estudios de meta-análisis han puesto de manifiesto la fiabilidad de las diferentes versiones de la prueba, entre ellas el RCADS-30, en distintos contextos, culturas e idiomas (Piqueras, Martín-Vivar, Sandín, San Luis, y Pineda, 2017). También se ha encontrado que la prueba es invariante en función del sexo, edad o método de aplicación (online vs lápiz y papel; Pineda, Martín-Vivar, Sandín, y Piqueras, 2017). La fiabilidad como coeficiente alfa obtenida con la presente muestra ha estado comprendida entre .64 y .90 : ansiedad de separación ( $\alpha=.69)$, fobia social $(\alpha=.79)$, trastorno de ansiedad generalizada $(\alpha=.82)$, trastorno de pánico $(\alpha=.72)$, trastorno obsesivo compulsivo $(\alpha=.70)$, trastorno depresivo mayor ( $\alpha$ $=.64)$ y puntuación total $(\alpha=.90)$.

Alabama Pareting Questionnaire for Children. El Alabama Parenting Questionnaire for Children (APQ; Frick, 1991; Shelton, Frick, y Wootton, 1996) es un cuestionario autoinformado de 42 ítems que mide 6 dimensiones relacionadas con la crianza, a saber: implicación parental (9 ítems para cada progenitor y 1 ítem común para ambos), crianza positiva (6 ítems), disciplina apropiada (6 ítems), disciplina inconsistente (6 ítems), pobre supervisión (10 ítems) y disciplina severa (4 ítems). A excepción de la escala de implicación parental que mide de forma separada la implicación en la crianza de cada progenitor, las escalas recogen información sobre la percepción del niño de las prácticas de sus padres sin distinguir entre ambos. Los ítems se contestan según una escala Likert de 5 puntos desde 1 (nunca) hasta 5 (siempre). El cuestionario APQ ha mostrado propiedades psicométricas adecuadas en distintas lenguas y comunidades (Cova et al., 2017; de la Osa, Granero, Penelo, Domè- 
nech, y Ezpeleta, 2014; Escribano, Aniorte, y Orgilés, 2013; Essau, Sasagawa, y Frick, 2006; Zlomke et al., 2014). En este trabajo aplicamos la versión española (Escribano et al., 2013; Servera, 2007). La consistencia interna de las subescalas para la presente muestra han sido las siguientes: implicación parental paterna $(\alpha=.82)$, implicación parental materna ( $\alpha$ $=.81)$, crianza positiva $(\alpha=.81)$, disciplina apropiada $(\alpha=.48)$, disciplina inconsistente $(\alpha$ $=.48$ ), pobre supervisión $(\alpha=.68)$ y disciplina severa $(\alpha=.71)$. Los coeficientes obtenidos han sido similares a los encontrados por los autores de la prueba, a excepción de la escala de disciplina inconsistente donde obtuvieron resultados ligeramente superiores $(\alpha=.67)$ y disciplina severa, donde parece que nuestra muestra presenta peores resultados $(\alpha=.46)$. En el trabajo de validación los autores no informaron sobre la consistencia de la escala de disciplina apropiada, que en el estudio original la nombraban como "otras disciplinas".

\section{Análisis estadístico}

Se hicieron una serie de análisis exploratorios de los datos con objeto de descartar la posible existencia de valores perdidos o patrones no esperados. A continuación se procedió a calcular el coeficiente de fiabilidad de cada una de las escalas aplicadas en el estudio. La finalidad de estos cálculos no es otra que la de garantizar que el grado de precisión de la medidas obtenidas en nuestra muestra fuera adecuado. Seguidamente y con la intención de explorar la estructura de las relaciones existentes, se realizó un análisis de las correlaciones entre todas las variables implicadas en el modelo. Posteriormente, para contrastar la hipótesis de las diferencias de edad y sexo, se realizó un análisis de varianza. Por último, y teniendo en cuenta los resultados del análisis correlacional, se llevaron a cabo una serie de análisis de regresión jerárquica por pasos, en los cuales se introdujeron en un primer paso las variables sociodemográficas y en un segundo las puntuaciones de las seis subescalas del APQ como variables predictoras y cada una de las escalas de síntomas de la RCADS como variables criterio. Los análisis se realizaron con el paquete estadístico SPSS 24 para Mac.

\section{Resultados}

Los resultados del análisis exploratorio no muestran valores destacables, las pruebas de asimetría y curtosis ha dado resultados aceptables, no hay presencia de valores extremos. Los resultados del análisis de fiabilidad de las escalas han resultado adecuados a excepción de las escalas de disciplina inconsistente $(\alpha=.48)$ y disciplina apropiada $(\alpha=.48)$. Los estadísticos descriptivos de las escalas, así como los resultados del análisis de varianza pueden verse en la Tabla 1. En el caso de las escalas de sintomatología, se han encontrado diferencias estadísticamente significativas para los síntomas de depresión y de ansiedad por separación en función de la edad de los sujetos. Para ambas escalas se cumple que a mayor edad de los sujetos, menor es la sintomatología informada. También encontramos diferencias similares y en el mismo sentidos para la escala de pobre supervisión del APQ. No se han encontrado diferencias en cuanto la sintomatología informada en función del sexo de los participantes. Sin embargo, parece que si hay diferencias en lo relativo a los hábitos de crianza de los padres en función del sexo de los hijos. En este sentido, los resultados muestran diferencias estadísticamente significativas en la implicación de la madre, crianza positiva, disciplina apropiada, pobre supervisión y disciplina severa. 
Tabla 1. Estadísticos descriptivos y diferencias entre grupos de edad y sexo

\begin{tabular}{|c|c|c|c|c|c|c|c|}
\hline & $\begin{array}{c}7-8 \text { años } \\
n=29\end{array}$ & $\begin{array}{c}9-10 \text { años } \\
n=129\end{array}$ & $\begin{array}{c}11-12 \text { años } \\
n=53\end{array}$ & $\begin{array}{c}\text { ANOVA } \\
F(2,210)\end{array}$ & $\begin{array}{l}\text { Chicas } \\
n=93\end{array}$ & $\begin{array}{l}\text { Chicos } \\
n=118\end{array}$ & $\begin{array}{c}\text { ANOVA } \\
F(1,210) \\
\end{array}$ \\
\hline Síntomas depresivos ${ }^{\mathrm{a}}$ & $2.69(1.13)$ & $1.87(1.55)$ & $1.26(1.70)$ & $7.42^{* * *}$ & $1.84(1.64)$ & $1.81(1.68)$ & 0.07 \\
\hline Pánico & $2.00(1.89)$ & $1.76(2.06)$ & $1.34(1.63)$ & 1.23 & $1.56(1.72)$ & $1.77(2.09)$ & 0.61 \\
\hline Fobia social & $4.45(3.01)$ & $4.64(3.22)$ & $4.36(3.51)$ & 0.15 & $4.83(3.22)$ & $4.30(3.27)$ & 1.40 \\
\hline Ansiedad por separación & $3.86(3.36)$ & $3.59(2.81)$ & $1.98(1.86)$ & $7.69^{* * *}$ & $3.60(3.02)$ & $2.93(2.54)$ & 3.05 \\
\hline Ansiedad generalizada & $6.51(3.30)$ & $6.70(2.82)$ & $6.45(3.21)$ & 0.15 & $6.90(2.94)$ & $6.38(3.00)$ & 1.54 \\
\hline Obsesiones-compulsiones & $4.37(2.38)$ & $3.93(2.65)$ & $3.20(2.67)$ & 2.22 & $3.92(2.76)$ & $3.72(2.54)$ & 0.31 \\
\hline $\begin{array}{l}\text { Síntomas ansioso-depre- } \\
\text { sivos }\end{array}$ & $\begin{array}{c}23.90 \\
(11.92)\end{array}$ & $\begin{array}{c}22.50 \\
(10.90)\end{array}$ & $\begin{array}{c}18.60 \\
(10.44)\end{array}$ & $3.05^{*}$ & $\begin{array}{c}22.68 \\
(10.99)\end{array}$ & $\begin{array}{c}20.94 \\
(11.06)\end{array}$ & 1.31 \\
\hline Implicación de la madre ${ }^{b}$ & $25.72(6.28)$ & $26.56(7.64)$ & $27.2(7.16)$ & 0.39 & $\begin{array}{l}27.97 \\
(6.75)\end{array}$ & $\begin{array}{l}25.55 \\
(7.61)\end{array}$ & $5.92^{*}$ \\
\hline Implicación del padre & $24.72(7.32)$ & $23.28(8.21)$ & $\begin{array}{l}24.45 \\
(7.66)\end{array}$ & 0.66 & $\begin{array}{l}24.52 \\
(7.75)\end{array}$ & $\begin{array}{l}23.19 \\
(8.08)\end{array}$ & 1.48 \\
\hline Crianza positiva & $18.41(3.87)$ & $18.20(4.63)$ & $\begin{array}{l}17.30 \\
(5.51)\end{array}$ & 0.80 & $\begin{array}{l}18.92 \\
(3.90)\end{array}$ & $\begin{array}{l}17.30 \\
(5.26)\end{array}$ & $6.22 *$ \\
\hline Disciplina apropiada & $6.41(2.95)$ & $7.32(3.65)$ & $6.89(3.15)$ & 0.96 & $6.38(3.17)$ & $7.63(3.56)$ & $7.06^{* *}$ \\
\hline Disciplina inconsistente & $7.86(4.18)$ & $7.98(3.91)$ & $7.30(3.81)$ & 0.58 & $8.21(3.85)$ & $7.47(3.94)$ & 1.89 \\
\hline Pobre supervisión & $8.20(5.88)$ & $5.61(4.72)$ & $5.8(5.54)$ & $3.13^{*}$ & $5.21(4.70)$ & $6.61(5.42)$ & $3.93^{*}$ \\
\hline Disciplina severa & $3.06(2.52)$ & $2.92(2.65)$ & $2.85(2.90)$ & 0.06 & $2.30(2.12)$ & $3.40(2.97)$ & $9.18^{* *}$ \\
\hline
\end{tabular}

Nota $: \mathrm{a}=$ Prácticas de crianza medias con el APQ (Escribano et al., 2013); b = Síntomas ansioso-depresivos medidos con el RCADS-30 (Sandín et al., 2010). * $p<.05$.; ** $p<.01$; $* * * p<.001$.

Los resultados del análisis de correlaciones entre las variables se muestran en la Tabla 2. Puede apreciarse como la implicación materna se asocia positiva y significativamente con la sintomatología obsesiva-compulsiva. Sin embargo la implicación paterna se relaciona negativa y significativamente con los síntomas depresivos y de pánico. Parece por otro lado que la implicación de ambos progenitores está altamente relacionada y a su vez ambas correlacionan negativa y significativamente con la disciplina severa. La crianza positiva parece estar relacionada con la implicación de ambos progenitores. Sin embargo, esta crianza positiva parece estar relacionada también con la aparición de sintomatología obsesivacompulsiva y la sintomatología ansioso-depresiva en general. Otras prácticas educativas relacionadas positiva y significativamente con la sintomatología ansioso-depresiva en gen- 
eral, han sido las tres escalas de disciplina. Por otro lado, parece que la pobre supervisión se relaciona con los síntomas de depresión, pánico y obsesión-compulsión. A su vez la pobre supervisión parece estar estrechamente relacionada con todas las prácticas disciplinarias. Como se puede observar, las relaciones entre las prácticas de crianza y la sintomatología ansioso-depresiva son complejas. Para tratar de desgranar estas relaciones procedemos con los análisis de regresión jerárquica.

Para examinar la relación diferencial de las diferentes estrategias educativas y la sintomatología ansioso-depresiva se realizaron una serie de análisis de regresión jerárquica por pasos, en las que en el primer paso ese incluían como variables predictoras las demográficas y en un segundo paso el resto de variables. Solo se retuvieron aquellas variables predictoras que resultaron estadísticamente significativas en los análisis de correlación previos.

Los resultados de esta serie de análisis aparecen en la Tabla 3. Salvo los relativos a R2, el resto de valores de la tabla corresponden a los resultados obtenidos en el último paso del análisis, esto es, se refieren al momento en el que las variables predictoras estaban incluidas

Tabla 2. Correlaciones entre las variables relevantes en el modelo

\begin{tabular}{|c|c|c|c|c|c|c|c|}
\hline & $\begin{array}{l}\text { Implicación } \\
\text { de la madre }\end{array}$ & $\begin{array}{c}\text { Implicación } \\
\text { del padre }\end{array}$ & $\begin{array}{l}\text { Crianza } \\
\text { positiva }^{\text {a }}\end{array}$ & $\begin{array}{l}\text { Disciplina } \\
\text { apropiada }^{\mathrm{a}}\end{array}$ & $\begin{array}{l}\text { Disciplina in- } \\
\text { consistente }^{\mathrm{a}}\end{array}$ & $\begin{array}{l}\text { Pobre su- } \\
\text { pervisión }^{\mathrm{a}}\end{array}$ & $\begin{array}{c}\text { Disciplina } \\
\text { severa }^{\mathrm{a}}\end{array}$ \\
\hline Síntomas depresivos ${ }^{\mathrm{b}}$ & -.042 & $-143^{*}$ & -.11 & $.144^{*}$ & $.209^{* *}$ & $.268^{* * *}$ & $.270^{* * *}$ \\
\hline Pánico ${ }^{b}$ & -.027 & $-.150^{*}$ & -.086 & $.222^{* * *}$ & $.238^{* * *}$ & $.251^{* * *}$ & $.324^{* * *}$ \\
\hline Fobia social $^{\mathrm{b}}$ & .094 & -.059 & .091 & .128 & $.156^{*}$ & .031 & $.158^{*}$ \\
\hline Ansiedad por separación ${ }^{\mathrm{b}}$ & -.036 & -.024 & .086 & .112 & -.036 & -.058 & -.005 \\
\hline Ansiedad generalizada $^{\mathrm{b}}$ & .125 & .076 & $.196^{* *}$ & .084 & .039 & -.057 & -.002 \\
\hline Obsesiones-compulsiones ${ }^{\mathrm{b}}$ & $.152^{*}$ & .042 & $.137^{*}$ & $.198^{* *}$ & $.211^{* *}$ & $.157^{*}$ & .117 \\
\hline Síntomas ansioso-depresivos ${ }^{b}$ & .078 & -.041 & .102 & $.197^{* *}$ & $.171^{*}$ & .101 & $.170^{*}$ \\
\hline Implicación de la madre & - & $.702^{* * *}$ & $.687^{* * *}$ & -.003 & $.181^{* *}$ & -.034 & $-.190^{* *}$ \\
\hline Implicación del padre & & - & $.572^{* * *}$ & .077 & .010 & -.013 & $-.197^{* *}$ \\
\hline Crianza positiva & & & - & -.036 & .133 & -.027 & $-.173^{*}$ \\
\hline Disciplina apropiada & & & & - & .127 & $.175^{*}$ & $.305^{* * *}$ \\
\hline Disciplina inconsistente & & & & & - & $.373^{* * *}$ & $.204^{* *}$ \\
\hline Pobre supervisión & & & & & & - & $.337^{* * *}$ \\
\hline
\end{tabular}

Nota: $\mathrm{a}=$ Prácticas de crianza medias con el APQ (Escribano et al., 2013); $\mathrm{b}=$ Síntomas ansioso-depresivos medidos con el RCADS-30 (Sandín et al., 2010). $* x<.05 . ; * *<.01$;; $* * * p<.001$. 
Tabla 3. Análisis de regresión múltiple jerárquica y de pasos sucesivos: Efectos de las dimisiones de los estilos de crianza ${ }^{a}$ sobre la predicción de síntomas ansioso-depresivos ${ }^{\mathrm{b}}$

\begin{tabular}{|c|c|c|c|c|c|c|c|c|}
\hline Variable predictora & Variable criterio & $R^{2}$ & $\Delta R^{2}$ & $B$ & ET B & $\beta$ & $t$ & $r_{12.3}$ \\
\hline Edad & Depresión & .05 & .05 & -0.31 & 0.10 & -.19 & $-3,04^{* *}$ & -.21 \\
\hline Disciplina severa & & .12 & .07 & 0.13 & 0.04 & .22 & $3.09^{* * *}$ & .20 \\
\hline Pobre supervisión & & .11 & .03 & 0.06 & 0.02 & .19 & $2.76^{* *}$ & .18 \\
\hline Disciplina severa & Pánico & .11 & .11 & 0.21 & 0.05 & .29 & $4.34^{* * *}$ & .29 \\
\hline Disciplina inconsistente & & .14 & .03 & 0.09 & 0.03 & .18 & $2.67^{* *}$ & .18 \\
\hline Disciplina severa & Fobia Social & .03 & .03 & 0.19 & 0.08 & .16 & $2.32^{*}$ & .16 \\
\hline Edad & Ansiedad por separación & .08 & .09 & -0.77 & 0.18 & -.29 & $-4.35^{* * *}$ & -.29 \\
\hline Crianza positiva & Ansiedad generalizada & .04 & .04 & 0.12 & 0.04 & .20 & $2.89 * *$ & .20 \\
\hline Edad & Obsesión-compulsión & .03 & .03 & -0.43 & 0.17 & -.17 & $-2.59 * *$ & -.18 \\
\hline Disciplina apropiada & & .07 & .04 & 0.14 & 0.05 & .19 & $2.83^{* *}$ & .19 \\
\hline Disciplina inconsistente & & .10 & .03 & 0.11 & 0.05 & .16 & $2.38^{*}$ & .16 \\
\hline Implicación de la madre & & .12 & .02 & 0.05 & 0.02 & .13 & $2.00^{*}$ & .14 \\
\hline Edad & Ansioso-depresivos & .03 & .03 & -2.01 & 0.70 & -.19 & $-2.87^{* *}$ & -.20 \\
\hline Disciplina apropiada & & .08 & .04 & 0.60 & 0.21 & .19 & $2.83^{* *}$ & .19 \\
\hline Disciplina inconsistente & & .10 & .02 & 0.40 & 0.18 & .14 & $2.12^{*}$ & .15 \\
\hline
\end{tabular}

Nota $: \mathrm{a}=$ Prácticas de crianza medias con el APQ (Escribano et al., 2013); b = Síntomas ansioso-depresivos medidos con el RCADS-30 (Sandín et al., 2010). * $p<.05$. ; ** $p<.01$. ; $* * * p<.001$.

en la ecuación. Puede apreciarse como el sexo no ha resultado un predictor relevante para la sintomatología ansioso-depresiva. Por su parte la edad sí parece un buen predictor de la sintomatología ansioso-depresiva en general y más concretamente asociada a la depresión, ansiedad por separación y síntomas de obsesión-compulsión.

En cuanto a las estrategias educativas, la disciplina en cualquiera de las tres dimensiones que recoge el APQ, han resultado ser buenos predictores de los síntomas emocionales de los niños, llegando a explicar hasta el 14\% de la varianza en algunos casos.

Por último los resultados obtenidos apuntan a que la implicación maternal en la crianza parece ser un factor de riesgo para la aparición de síntomas de obsesión y compulsión. 


\section{Discusión}

Los objetivos de esta investigación han sido examinar la relación entre las prácticas educativas parentales y los síntomas interiorizados de los niños y si existen diferencias en función del sexo de los progenitores, por un lado y explorar el papel que la edad y el sexo de los niños pueden desempeñar en estas relaciones por otro.

En general, los datos obtenidos apoyan las hipótesis de los autores aunque con algunos matices. Aunque hemos encontrado diferencias de sexo en las prácticas educativas desarrolladas por los padres, el sexo de los niños no parece ser un buen predictor de los síntomas de ansiedad y depresión. Si bien algunos estudios previos han mostrado importantes diferencias en función del sexo (Mestre, Samper, Tur, y Díez, 2001), otros sin embargo no han podido encontrar estas diferencias (Musitu y Cava, 2003), parece por tanto que la relación entre el sexo de los menores y la percepción del estilo educativo de los padres puede resultar un tanto esquiva.

Por su parte, la edad sí ha resultado relevante en la predicción de síntomas, mostrado una asociación negativa y estadísticamente significativa con muchos de los trastornos. En general, los resultados reportados por la investigación, sostienen la idea de que, a medida que los niños se van desarrollando, perciben una disminución del apoyo e implicación de ambos padres. Estas diferencias bien podrían deberse al cambio efectivo de estrategias educativas por parte de los progenitores (Rodríguez, Barrio, y Carrasco, 2009). Sin embargo, nuestros resultados apuntan que también cambiaría la influencia que estos estilos tienen sobre los menores, con independencia que haya una evolución en la estrategia educativa de los padres. A la vista de estos resultados podemos considerar la edad como un factor de protección para la sintomatología ansioso-depresiva en general y más concretamente para la depresión, la ansiedad por separación y los síntomas de obsesión-compulsión asociados a las practicas parentales, esto es a mayor edad de los niños, menor efecto tienen estas prácticas en la sintomatología que presentan.

En lo relativo a la implicación educativa de los progenitores, y en concreto la implicación materna, lejos de ser un factor de protección, parece que puede estar relacionada con la aparición de sintomatología obsesiva-compulsiva. Esta relación diferencial de ambos progenitores ya había sido reportada con anterioridad, por ejemplo Conell y Goodman, en un trabajo de meta-análisis, han confirmado la presencia de esta asociación diferencial entre la psicopatología del padre/madre y los síntomas internalizados de los hijos (Connell y Goodman, 2002).

Los resultados del análisis de la fiabilidad del APQ para esta muestra parecen adecuados, aunque cabe decir que dos de ellas no alcanzarían valores de .60 que representa el valor mínimo exigible a una escala para considerarla suficientemente fiable como para usarla en una investigación (Nunnally, 1978). A la vista de estos resultados, pueden ser necesarios realizar nuevos trabajos de análisis factorial que mejoren, o en caso necesario incluso prescindan, de alguna de las escalas del APQ.

Alguna de las limitaciones de este trabajo ha sido el tamaño muestral, pues se trata de una muestra amplia, aunque no lo suficiente para asegurar la estabilidad de la matriz de co- 
rrelaciones. Otra de las limitaciones ha sido la selección de la muestra, dado que han sido participantes seleccionados de una provincia concreta y puede que los resultados no sean totalmente equiparables al resto de la población. También ha sido una limitación la transversalidad de los datos. Futuras investigaciones deberían explorar estos resultados con diseños de corte longitudinal, muestras más amplias y con mayor representatividad.

Para concluir, cabe destacar que estos resultados puede tener importantes implicaciones en la práctica clínica, por ejemplo a la hora de tomar decisiones sobre la custodia de un menor, parece que no debe considerarse una determinada conducta educativa en ambos progenitores por igual, puesto que la repercusión que puede tener en los menores parece ser distinta en función de si es el padre o la madre quien exhibe el comportamiento. Algo similar ocurre con la edad de los menores donde vemos como el estilo educativo de los progenitores va perdiendo relevancia.

\section{Referencias}

Baumrind, D. (1968). Authoritarian vs. authoritative parental control. Adolescence, 3(11), 255-272. Retrieved from http:// psycnet.apa.org/psycinfo/1969-06772-001

Chorpita, B. F., Yim, L., Moffit, C., Umemoto, L. A., y Francis, S. E. (2000). Assessment of DSM-IV anxiety and depression in children: A revised child anxiety and depression scale. Behaviour Research and Therapy, 38, 835-855.

Connell, A. M., y Goodman, S. H. (2002). The association between psychopathology in fathers versus mothers and children's internalizing and externalizing behavior problems: A meta-analysis. Psychological Bulletin, 128(5), 746-773. https://doi.org/10.1037/0033-2909.128.5.746

Cova, F., Bustos, C., Rincón, P., Streiner, D. L., Grandón, P., Saldivia, S., ... Contreras, G. (2017). Psychometric properties of the Alabama Parenting Questionnaire adapted to families of chilean preschoolers. Infant Mental Health Journal, 38(2), 249-257. https://doi.org/10.1002/imhj.21631

de la Osa, N., Granero, R., Penelo, E., Domènech, J. M., y Ezpeleta, L. (2014). Psychometric properties of the Alabama parenting questionnaire-preschool revision (APQ-Pr) in 3 year-old Spanish preschoolers. Journal of Child and Family Studies, 23(5), 776-784.

Escribano, S., Aniorte, J., y Orgilés, M. (2013). Estructura factorial y propiedades psicométricas de la versión española del Alabama Parenting Questionnaire (APQ) para niños. Psicothema, 25(3), 324-329. https://doi.org/10.7334/ psicothema2012.315

Essau, C., Sasagawa, S., y Frick, P. J. (2006). Psychometric properties of the Alabama Parenting Questionnaire. Journal of Child and Family Studies, 15(5), 597-616.

Frick, P. (1991). The Alabama Parenting Questionnaire. Unpublished rating scale, University of Alabama, 223-. Retrieved from http://scholar.google.co.uk/scholar?q=frick+and+alabama+parenting+questionnaireybtnG=yh I=enyas_sdt=0,5\#0

Maccoby, E. E., y Martin, J. A. (1983). Socialization in the context of the family: Parent-child interaction. En P. H. Mussen (Ed.) Handbook of child psichology: Vol. 4. Socialization, personality, and social development (pp. 1-102).

Mestre, M., Samper, P., Tur, A., y Díez, I. (2001). Estilos de crianza y desarrollo prosocial de los hijos. Revista de Psicología General y Aplicada, 54(4), 691-703. https://doi.org/10.1016/j.gnr.2010.03.001

Musitu, G., y Cava, J. (2003). El rol del apoyo social en el ajuste de los adolescentes. Intervevncion Psicosocial, 12(2), 179-192.

Nunnally, J. C. (1978). Psychometric Theory. rdsepiucsforg (Vol. 3). Retrieved from http://rds.epi-ucsf.org/ticr/syllabus/courses/46/2005/10/20/Lecture/readings/Psychometric Theory.pdf 
Ordóñez-Ortega, A., Espinosa-Fernández, L., García-López, L. J., y Muela-Martínez, J. A. (2013). Inhibición conductual y su relación con los trastornos de ansiedad infantil. Terapia Psicologica, 31(3), 355-362. https://doi. org/10.4067/S0718-48082013000300010

Pineda, D., Martín-Vivar, M., Sandín, B., y Piqueras, J. A. (2017). Factorial invariance and norms of the 30-item reduced version of the Revised Child Anxiety and Depression Scale. Manuscrito en revisión.

Piqueras, J. A., Martín-Vivar, M., Sandín, B., San Luis, C., y Pineda, D. (2017). The Revised Child Anxiety and Depression Scale: A systematic review and reliability generalization meta-analysis. Journal of Affective Disorders, 218(15), 153-169. https://doi.org/10.1016/j.jad.2017.04.022

Rodríguez, M. Á., Barrio, M. V. Del, y Carrasco, M. Á. (2009). ¿Cómo perciben los hijos la crianza materna y paterna? Diferencias por edad y sexo. Escritos de Psicología, 2(2), 10-18.

Romano, E., Tremblay, R. E., Boulerice, B., y Swisher, R. (2005). Multilevel correlates of childhood physical aggression and prosocial behavior. Journal of Abnormal Child Psychology, 33(5), 565-578. https://doi.org/10.1007/ s10802-005-6738-3

Rutter, M. (2003). Genetic influences on risk and protection: Implications for understanding resilience. En S. S. Luthar (Ed.), Resilience and vulnerability: Adaptation in the context of childhood adversities (pp. 489509). Cambridge University Press.

Sandin, B., Chorot, P., Valiente, R. M., y Chorpita, B. F. (2010). Development of a 30-item version of the Revised Child Anxiety And Depression Scale. Revista de Psicopatología Y Psicología Clínica, 15(3), 165-178. https://doi. org/10.5944/rppc.vol.15.num.3.2010.4095

Sandín, B., Valiente, R. M., y Chorot, P. (2009). RCADS : evaluación de los síntomas de los trastornos de ansiedad y depresión en niños y adolecentes. Revista de Psicopatología Y Psicología Clínica, 14(3), 193-206. https://doi. org/http://dx.doi.org/10.5944/rppc.vol.14.num.3.2009.4078

Servera, M. (2007). Versión en español del Alabama Parenting Questionnaire (APQ), versión para niños [Spanish version of the Alabama Parenting Questionnaire (APQ) for children]. Unpublished document.

Shelton, K. K., Frick, P. J., y Wootton, J. (1996). Assessment of parenting practices in families of elementary schoolage children. Journal of Clinical Child Psychology, 25(3), 317-329. https://doi.org/10.1207/s15374424jccp2503_8

Spence, S. H. (1997). The structure of anxiety symptoms among children: a confirmatory factor analytic study. Journal of Abnormal Psychology, 106, 280-297.

Spence, S. H. (1998). A measure of anxiety symptoms among children. Behaviour Research and Therapy, 36(5), 545-566. https://doi.org/10.1016/S0005-7967(98)00034-5

Tur, A. M., Mestre, M. V., y del Barrio, M. V. (2014). Factores moduladores de la conducta agresiva y prosocial. El efecto de los hábitos de crianza en la conducta del adolescente. Ansiedad Y Estrés, 10(1), 75-88.

Valiente, R. M., Sandín, B., y Chorot, P. (2012). El miedo en niños y adolescentes. Crítica, 62(977), 23-27.

Vostanis, P., Graves, A., Meltzer, H., Goodman, R., Jenkins, R., y Brugha, T. (2006). Relationship between parental psychopathology, parenting strategies and child mental health: Findings from the GB national study. Social Psychiatry and Psychiatric Epidemiology, 41(7), 509-514. https://doi.org/10.1007/s00127-006-0061-3

Zlomke, K. R., Lamport, D., Bauman, S., Garland, B., y Talbot, B. (2014). Parenting Adolescents: Examining the Factor Structure of the Alabama Parenting Questionnaire for Adolescents. Journal of Child and Family Studies, 23(8), 1484-1490. https://doi.org/10.1007/s10826-013-9803-5 